\title{
Restenosis Rates After Carotid Endarterectomy with Primary Closure Under Regional Anesthesia: Results of a Single Center Study with 553 Patients
}

\section{(DI İbrahim Erdinç1, (1) Fulya Yılmaz², (1) Koray Baş3}

${ }_{1}^{1}$ University of Health Sciences Turkey, İzmir Bozyaka Training and Research Hospital, Department of Cardiovascular Surgery, İzmir, Turkey 2University of Health Sciences Turkey, İzmir Bozyaka Training and Research Hospital, Department of Anesthesiology and Reanimation, İzmir, Turkey

3̇̇zmir Bakırçay University Faculty of Medicine, Department of General Surgery, İzmir, Turkey

\begin{abstract}
Objectives: Carotid endarterectomy (CEA) is the current gold standard management for carotid artery stenosis but there is still a debate on which closure technique is superior to lower postoperative restenosis rates. The aim of this retrospective study is to assess "restenosis rates" of our 553 patients who underwent CEA "under regional anesthesia" with "primary closure" technique.

Materials and Methods: We retrospectively evaluated patients who underwent CEA, by non-shunting technique under regional anesthesia, with primary longitudinal arteriotomy closure between 2008 and 2019.

Results: Five hundred and fifty three patients (409 male and 144 female) were evaluated. There were no statistically significant differences in terms of demographic characteristics, sides of stenosis, operation time, preoperative stenosis ratio, and postoperative hospital stay between the gender groups. None of the patients developed restenosis (stenosis rate of over $50 \%$ ) after primary closure under regional anesthesia during the two-year follow-up period.
\end{abstract}

Address for Correspondence: Fulya Y1lmaz, University of Health Sciences Turkey, İzmir Bozyaka Training and Research Hospital, Department of Anesthesiology and Reanimation, İzmir, Turkey e-mail: fulya.dr@gmail.com ORCID: orcid.org/0000-0002-6901-7404

Received: 12.06.2021 Accepted: 15.10.2021

Cite this article as: Erdinç E, Yılmaz F, Baş K. Restenosis Rates After Carotid Endarterectomy with Primary Closure Under Regional Anesthesia: Results of a Single Center Study with 553 Patients. EJCM 2021;9(4):178-183.

DOI: 10.32596/ejcm.galenos.2021-06-035 
Conclusion: According to our results, CEA with "primary closure under regional anesthesia" is a safe and effective surgical treatment for carotid stenosis without postoperative restenosis. We thought that further studies, which investigate the rate of restenosis after CEA, should also evaluate the "type of the anesthesia management" as a factor on it.

Keywords: Carotid artery stenosis, carotid endarterectomy, primary longitudinal arteriotomy closure, patch closure, postoperative restenosis, cervical plexus block

\section{Introduction}

Carotid endarterectomy (CEA) is the current gold standard management in reducing the risk of stroke in both symptomatic (significant stenosis $>70 \%$ ) and well selected asymptomatic patients with internal artery stenosis $^{(1-10)}$. Currently, there are two surgical approaches in the literature: Traditional endarterectomy with longitudinal arteriotomy and the eversion technique ${ }^{(3)}$. In terms of closure techniques after CEA, there are also two options: primary closure (PRC) and patch closure (PAC) by saphenous vein or a synthetic material ${ }^{(3,6)}$. While a theoretical advantage of PAC is the reduction of the rate of re-stenosis, its disadvantages include longer carotid cross-clamping time and possible morbidities associated with vein harvesting including hemorrhage and/or infection on surgical site. On the other hand, PRC and proper medical management may be equally effective in preventing recurrent stenosis and has the advantage of reduced operative time ${ }^{(1)}$.

The aim of this retrospective study is to assess restenosis rates of patients who underwent CEA under regional anesthesia with primary closure between 2008 and 2019.

\section{Materials and Methods}

We retrospectively evaluated 553 patients, who underwent CEA by non-shunting technique under regional anesthesia with primary-longitudinal arteriotomy- closure due to carotid artery stenosis between 2008 and 2019, in terms of demographic characteristics, co-morbidities, preoperative and postoperative stenosis ratio, operation time, hospital stay, and post-CEA restenosis.

\section{Patient Selection for Operation and Management of Postoperative Anticoagulant Treatment in Our Institution}

In more than $70 \%$ of asymptomatic patients and over $60 \%$ of symptomatic patients, stenosis was determined as an indication for surgery. In patients with bilateral carotid artery stenosis, symptomatic side was firstly operated in symptomatic patients and the side with higher grade stenosis was firstly operated in asymptomatic patients was. In asymptomatic patients, their medical treatment was started after the diagnosis and they were operated as soon as possible. On the other hand, symptomatic patients were operated within 15 days after the diagnosis under medical therapy. If the patients had high grade stenosis or neurologic deterioration due to cerebral hypoperfusion, they were operated urgently.

Since the patients were awake and conscious in the early postoperative period, after control of bleeding and hematoma within 2 hours after the surgery, clopidogrel $75 \mathrm{mg}$ and acetylsalicylic acid $100 \mathrm{mg}$ were given per oral, and heparin infusion (500 IU/hour) was started and continued approximately for 6-7 hours. Then, patients were evaluated again for bleeding and clopidogrel $75 \mathrm{mg}$ and acetylsalicylic acid $100 \mathrm{mg}$ heparin was increased to 7500-1000 IU and continued for 18-24 hours.

\section{Anesthetic Management}

No premedication was administered on the day of the surgery. In the operating room, a peripheral venous line was established and a standard monitorization, including a peripheral pulse-oximetry, a 3-lead electrocardiography 
and a contralateral intra-arterial blood pressure monitoring, was applied.

\section{Deep Cervical Plexus Block (C2-C3-C4) combined with Superficial Cervical Plexus Block}

The anatomical landmarks were identified and marked on the skin as sternocleidomastoid muscle (SCM), cricoid cartilage, and mastoid process. After skin preparation, deep cervical plexus blocks were performed with $5 \mathrm{~mL}$ of the combination of $2.5 \mathrm{~mL}$ of $0.5 \%$ bupivacaine and 2.5 $\mathrm{mL}$ of $2 \%$ prilocaine near each sensory branch of nerve roots of $\mathrm{C}_{2}, \mathrm{C}_{3}, \mathrm{C}_{4}$. Then, superficial cervical plexus block by $10 \mathrm{~mL}$ of the combination of $5 \mathrm{~mL} 0.5 \%$ bupivacaine and $5 \mathrm{~mL} \mathrm{2 \%}$ prilocaine was applied at the level of the $6^{\text {th }}$ cervical vertebra by conventional technique.

\section{Surgical Technique}

After performing a parallel incision to SCM, the External Carotid Artery (ECA) and Common Carotid Artery (CCA) were dissected and suspended by tapes. Then, patient was given 5000 IU heparin intravenously before clamping of the carotid artery. After clamping of the artery, consciousness and motor status of patient were tested. If there was no change in the status of patient, endarterectomy was performed. At the end of the procedure, the incision was closed primarily with 6-0 polypropylene suture.

\section{Follow-up}

Patients were regularly followed up in outpatient clinics every 6 months for two years after the operation. Carotid duplex ultrasound was performed in every followup visit on all patients. Carotid stenosis ratio over 50\% was accepted as "restenosis" after CEA surgery.

\section{Statistical Analysis}

Statistical data analysis was performed by SPSS version 21 software (SPSS, Inc., Chicago, IL, USA). Numerical data were given as mean \pm standard deviation and were analyzed using the Student's t-test. $p<0.05$ was considered statistically significant.

\section{Results}

Five hundred and fifty-three patients underwent unilateral CEA due to unilateral or bilateral carotid artery stenosis by the same surgery and anesthesia team between 2008 and 2019. The rates of male and female patients were $74 \%(n=409)$ and $26 \%(n=144)$, respectively.

Preoperative duplex ultrasound evaluations of patients revealed that there was stenosis on the left side in 282 patients (211 male, 70 female) and on the right side in 272 patients (198 male, 74 female). Demographic characteristics, preoperative stenosis ratio, perioperative surgical time and postoperative hospital stay are shown in Table 1. Comorbidities of patients according to gender is shown in Table 2 . There were no statistically significant

Table 1. Demographic characteristics, preoperative stenosis ratio, perioperative surgical time and postoperative hospital stay of the patients

\begin{tabular}{|l|l|l|l|}
\hline & Male $(\mathbf{n = 4 0 9 )}$ & Female $(\mathbf{n = 1 4 4 )}$ & p-value \\
\hline Age & $67.04 \pm 8.76$ & $67.88 \pm 9.40$ & 0.612 \\
\hline Preoperative stenosis rate (\%) & $76.31 \pm 10.19$ & $75.89 \pm 10.25$ & 0.957 \\
\hline Surgical time (min) & $58.78 \pm 12.26$ & $60.99 \pm 12.69$ & 0.193 \\
\hline Post-operative hospital-stay (day) & $3.55 \pm 1.57$ & $3.45 \pm 1.70$ & 0.422 \\
\hline
\end{tabular}

Table 2. Comorbidities of the patients according to gender

\begin{tabular}{|l|l|l|l|l|}
\hline & Hypertension & Hyperlipidemia & Diabetes mellitus & Smoking \\
\hline Male $(\mathbf{n = 4 0 9 )}$ & 318 & 325 & 292 & 306 \\
\hline Female $(\mathbf{n = 1 4 4 )}$ & 97 & 110 & 52 & 85 \\
\hline
\end{tabular}


differences in terms of demographic characteristics, operation time, preoperative stenosis ratio, and postoperative hospital stay between the gender groups.

After the administration of systemic IV heparin (5000 IU), distal part of the ICA was subsequently clamped and neurological examination was performed. Consciousness, orientation, and cooperation with verbal stimuli were evaluated in patients under regional anesthesia. The patient was asked to move the contralateral upper and lower extremities, and the neurological examination was continued for 2-3 minutes ${ }^{(11)}$. Five patients required intraoperative shunt insertions due to immediate deterioration of the consciousness after carotid clamping. Three patients developed localized hematoma after the surgery that necessitated decompression surgeries.

No mortality and mortality were noticed due to operation in postoperative period. Five patients that required intraoperative shunt insertions during the surgery were evaluated by neurology doctors postoperatively. No permanent sequelae were observed in patients and they were advised to continue the appropriate medical treatments. None of the patients developed restenosis (stenosis rate over 50\%) during the two-year follow-up period after the surgery.

\section{Discussion}

Although many studies have demonstrated that CEA is efficient to prevent stroke in symptomatic and wellselected asymptomatic patients, there is still a debate on which closure technique is superior for CEA to lower postoperative restenosis rates ${ }^{(1-6)}$. Restenosis is defined as recurrent luminal narrowing of more than $50 \%$ after surgery ${ }^{(7,9,10)}$. It can be detected by Duplex ultrasound ${ }^{(9,10)}$, computed tomographic angiography, magnetic resonance angiography or conventional angiography. Duplex ultrasound scanning remains the most preferred diagnostic modality in clinical practice for screening ${ }^{(9)}$. After CEA procedure, the restenosis in the first year is mainly due to an intimal hyperplasia and later than 24 months, the progression of underlying atherosclerotic disease is a main reason ${ }^{(5,7,8,12)}$.

Restenosis ratio after CEA with primary closure has been documented to vary in $1 \%-36 \%$ in the literature li-4,7) $^{(1-3)}$ . Using patch angioplasty in CEA is suggested to reduce both risks of restenosis and recurrent ipsilateral stroke ${ }^{(3)}$.

Although surgical techniques such as PAC after conventional CEA have been shown by level-I evidence to decrease the incidence of restenosis after CEA, surgeons are still unwilling to adopt PAC for their routine use. How widely PAC is used in vascular surgery practice in the real-world is unknown $(2010)^{(12)}$. All the studies included in these reports were performed over 20 years ago and quality of trials was generally poor ${ }^{(4)}$. Although previous studies disfavor the use of PRC for CEA because of neurologic events and restenosis rates, recent studies have changed the approach on this issue $(2016)^{(6)}$.

Not only surgical technique but also other unknown factors may affect the restenosis in CEA. Lammeren et al. ${ }^{(10)}$ reported that asymptomatic patients had an increased risk for restenosis in the first year after CEA, compared to patients with either transient ischemic stroke or stroke. Also, they reported that early intervention (CEA within 30 days after stroke) was associated with decreased risk of restenosis. Garzon-Muvdi et al. ${ }^{(5)}$ reported that although multiple factors might contribute to restenosis after CEA, the important factor that predisposed patients to restenosis after CEA was a family history of stroke. Bonati et al. ${ }^{(13)}$ evaluated the restenosis and risk of stroke after stenting or endarterectomy. They reported that restenosis occurred more frequently after stenting than endarterectomy and increased the risk of ipsilateral stroke in the overall population.

Avgerinos et al. ${ }^{(6)}$ compared perioperative and longterm results of different CEA closure techniques. They reported that PRC could be performed with equivalent results to other techniques. Cheng et al..$^{(1)}$ reported that PRC for CEA was related to low rates of restenosis and effective to prevent stroke in short terms. They also 
reported that PRC had the advantage of reducing crossclamp times and eliminating graft specific complications when compared to PAC. On the other hand, Huizing's reports were not compatible with Cheng's reports.

Huizing et al. ${ }^{(4)}$ evaluated PRC and PAC in CEA for symptomatic carotid artery stenosis. They reported that selective patching could be recommended instead of routine patching for patients based on internal carotid artery diameter and other patient characteristics. Huizing et al. ${ }^{(2)}$ showed that long term re-stenosis was significantly higher in PRC than in PAC in their review.

In this study, we investigated the restenosis rates of our cases by non-invasive duplex ultrasound. We performed all CEA operations under combined superficial and deep cervical plexus blocks applied by conventional method.

To the best of our knowledge, a few studies have indicated the effect of anesthesia management type on the rate of restenosis after CEA procedure. Kim et al. ${ }^{(14)}$ assessed the feasibility and benefits of CEA under regional anesthesia versus general anesthesia in terms of the anesthesia method, neurological monitoring, shunt usage, and closure technique. They demonstrated that no carotid artery stenosis occurred after surgery under regional anesthesia with primary closure. Similarly, it might be claimed that performing our surgeries "under regional anesthesia" may be a factor to reduce the restenosis rates in our study. In addition, as van Lammeren et al. ${ }^{(10)}$ stated, performing the surgeries on the early phase of stroke, as in our institution, may be another factor that affects our results.

\section{Conclusion}

According to our results, CEA with "primary closure under regional anesthesia" is a safe and effective surgical treatment for carotid stenosis without postoperative restenosis. We think that further studies which investigates the rate of restenosis after CEA should also evaluate the "type of the anesthesia management" as a factor on it.-

\section{Ethics}

Ethics Committee Approval: Since this retrospective study was conducted before January 2020, ethics committee approval is not required. Hospital management data usage permission certificate is available.

Informed Consent: Retrospective study.

Peer-review: Externally peer-reviewed.

\section{Authorship Contributions}

Surgical and Medical Practices: İ.E., F.Y., Concept: F.Y., K.B., Design: İ.E., F.Y., Analysis and/or Interpretation: F.Y., K.B., Literature Search: F.Y., Writing: F.Y., K.B.

Conflict of Interest: The authors declare that they have no conflict of interest.

Financial Disclosure: The authors declare no financial support by any grant or research sponsor and no competing financial interest.

\section{References}

1. Cheng I, Vyas KS, Velaga S, Davenport DL, Saha SP. Outcomes of carotid endarterectomy with primary closure. Int J Angiol 2017;26:83-8.

2. Huizing E, Vos CG, van den Akker PJ, Schreve MA, de Borst GJ, Ünlü Ç. A systematic review of patch angioplasty versus primary closure for carotid endarterectomy. J Vasc Surg 2019;69:1962-74.e4.

3. Marsman MS, Wetterslev J, Jahrome AK, et al. Carotid endarterectomy with primary closure versus patch angioplasty in patients with symptomatic and significant stenosis: protocol for a systematic review with metaanalyses and trial sequential analysis of randomised clinical trials. BMJ Open 2019;9:e026419.

4. Huizing E, Vos CG, Hulsebos RG, van den Akker PJ, Borst GJ, Ünlü Ç. Patch angioplasty or primary closure following carotid endarterectomy for symptomatic carotid artery stenosis. Surg J (NY) 2018;4e96-e101.

5. Garzon-Muvdi T, Yang W, Rong X, et al. Restenosis after carotid endarterectomy: insight into risk factors and modification of postoperative management. World Neurosurg 2016;89:159-67.

6. Avgerinos ED, Chaer RA, Naddaf A, El-Shazly OM, Marone L, Makaroun MS. Primary closure after carotid endarterectomy is not inferior to other closure techniques. J Vasc Surg 2016;64:678-83.e1.

7. Texakalidis P, Giannopoulos S, Jonnalagadda AK, et al. Carotid artery endarterectomy versus carotid artery stenting for restenosis after carotid artery endarterectomy: a systematic review and meta-analysis. World Neurosurg 2018;115:421-9.e1.

8. Oszkinis G, Pukacki F, Juszkat R, et al. Restenosis after carotid endarterectomy: incidence and endovascular management. Interv Neuroradiol 2007;13:345-52. 
9. De Witte CJ, Lammeren GW, Moll FL, Borst GJ. Does restenosis still hamper the benefit of carotid artery revascularization? ISRN Vascular Medicine 2013. P. 1-5.

10. van Lammeren GW, Peeters W, de Vries JP, et al. Restenosis after carotid surgery: the importance of clinical presentation and preoperative timing. Stroke 2011;42:965-71.

11. Ugurlucan M, Filik ME, Caglar IM, et al. Carotid endarterectomy using a "home-constructed" shunt for patients intolerant to cross-clamping. Surg Today 2015;45:284-9.

12. Goodney PP, Nolan BW, Eldrup-Jorgensen J, Likosky DS, Cronenwett JL; Vascular Study Group of Northern New England. Restenosis after carotid endarterectomy in a multicenter regional registry. J Vasc Surg 2010;52:897904.

13. Bonati LH, Gregson J, Dobson J, et al. Restenosis and risk of stroke after stenting or endarterectomy for symptomatic carotid stenosis in the International Carotid Stenting Study (ICSS): secondary analysis of a randomised trial. Lancet Neurol 2018;17:587-96.

14. Kim JW, Huh U, Song S, Sung SM, Hong JM, Cho A. Outcomes of Carotid Endarterectomy according to the Anesthetic Method: General versus Regional Anesthesia. Korean J Thorac Cardiovasc Surg 2019;52:392-9. 